\title{
Low-Dose Ritonavir-Boosted Atazanavir (200/100 mg) Maintained a High Virological Efficacy Up To 4 Years in Treatment-Experienced HIV-1 Infected Adults: A Prospective Cohort Study from Asia
}

\author{
Anchalee Avihingsanon ${ }^{1,2, *}$, Tanakorn Apornpong ${ }^{1}$, Stephen J Kerr ${ }^{1,3}$, Wirach Make-a-nantawat', Narukjaporn Thammajaruk ${ }^{1,}$ Supalak \\ Phonphithak ${ }^{1}$, Reshmie A Ramautarsing ${ }^{1,4}$, Amanda Clarke ${ }^{1,3}$, Praphan Phanuphak ${ }^{1}$, David M Burger ${ }^{5}$, Kiat Ruxrungtham ${ }^{1,2}$ and HIV-NAT \\ 006 Study Team \\ ${ }^{1}$ HIV-NAT, Thai Red Cross AIDS Research Centre, Bangkok, Thailand \\ ${ }^{2}$ Department of Medicine, Faculty of Medicine, Chulalongkorn University, Bangkok, Thailand \\ ${ }^{3}$ Kirby Institute for Infection and Immunity in Society, University of New South Wales, Sydney, Australia \\ ${ }^{4}$ Department of Global Health, Academic Medical Center, University of Amsterdam, Amsterdam Institute for Global Health and Development (AlGHD), Amsterdam, The \\ Netherlands
}

${ }^{5}$ Radboud Institute for Health Sciences (RIHS), Nijmegen, The Netherlands

\begin{abstract}
Background: HIV requires lifelong treatment so ARV dose optimization is important for long-term efficacy and safety. Previously, low-dose atazanavir (ATV)/ritonavir(r) plus 2 NRTIs in HIV-infected Thai patients provided adequate plasma ATV concentrations and reduced risk of hyperbilirubinemia. However, long-term efficacy and safety data is limited.
\end{abstract}

Methods: $127 \mathrm{HIV-infected} \mathrm{adults} \mathrm{on} \mathrm{ATV/r200/100mg} \mathrm{plus} 2$ NRTIs were prospectively followed in Thailand. CD4 cell counts, HIV-RNA, and safety parameters were performed every 6 months. Estimated glomerular filtration rate (eGFR) was calculated by chronic kidney disease epidemiology (CKD-EPI). ATV plasma concentrations (ATV $\mathrm{C}_{\text {trough }}$ ) were assessed.

Results: Median body weight was $60 \mathrm{~kg}$ and $50 \%$ were females. Previous regimens were mainly standard dose ATV/r (54\%) and lopinavir/r (29\%). 93\% of them were on tenofovir and $9.4 \%$ had HIV-RNA > 50 copies $/ \mathrm{mL}$ at the time when lower dose of ATV/r was initiated. The median duration of lower dose ATV/r was 154 (IQR 65-271) weeks and $50.4 \%$ had been followed for more than 3 years. HIV-RNA $<50$ copies $/ \mathrm{mL}$ by on-treatment and intention-to-treat analysis were $91.2 \%$ and $81.8 \%$, respectively. Bilirubin and eGFR significantly improved $\left[83\right.$ to $88 \mathrm{ml} / \mathrm{min} / 1.73 \mathrm{~m}^{2}$ $(p<0.001)]$. Premature discontinuation occurred in $13(10.2 \%)$ patients due to toxicity (CKD, renal stone, hepatitis, pancreatitis) and death (sepsis, cerebral aneurysm, CKD). All 42 cases with available ATV $C_{\text {trough }}$ had adequate ATV concentrations of $>0.15 \mathrm{mg} / \mathrm{L}$.

Conclusions: Long-term use of ATV/r $200 \mathrm{mg} / 100 \mathrm{mg}$ based-HAART as a maintenance therapy for patients who are sensitive to PI was efficacious and well-tolerated. Use of low-dose ATV/r could benefit resource limited settings because it will reduce toxicity, increase accessibility and save costs.

Keywords: Low dose atazanavir/ritonavir; Plasma ATV concentrations; HIV-infected patients; Thailand

\section{Introduction}

Atazanavir (ATV) is one of the preferred protease inhibitors (PIs) for once-daily (QD) administration. It is currently used at a dose of either $300 \mathrm{mg}$ in combination with low-dose ritonavir (100mg) QD or, less frequently, at $400 \mathrm{mg}$ QD (without ritonavir), both taken with food [1]. ATV's major advantages are its simplicity of once-daily administration, low pill burden, minimal effect on lipid profiles, distinct resistance profile [2] and good efficacy when compared to boosted lopinavir (LPV/r) [3-5] or efavirenz [6]. However, the drug is relatively expensive and severe hyperbilirubinemia can occur in some patients. In addition, evidence suggests that renal stones $[7,8]$ and chronic kidney disease [9] are associated with the use of standard dose of ATV/r based regimen which is similar to that of boosted indinavir (IDV/r) administered 800/100 twice daily. It was shown that both IDV and ATV-related hyperbilirubinemia and renal stones are concentration dependent [10,11]. When lower dose of IDV/r 400/100 mg twice daily was used, its efficacy was not inferior to the standard dose [1216]. Recently, a low-dose randomized control trial of efavirenz 400 $\mathrm{mg}$, conducted in multinational sites, showed comparable efficacy and fewer toxicities compared to efavirenz at $600 \mathrm{mg}$ [17]. Based on these studies, we speculate that ATV/r may behave the same way too. When a retrospective cohort study conducted in Thai patients [18] showed that ATV may be efficacious when dosed at $200 \mathrm{mg}$ with ritonavir $100 \mathrm{mg}$ QD, we investigated this and found that the pharmacokinetic parameters of ATV/r 200/100 mg QD plus 2 nucleos(t)ide reverse transcriptase(NRTIs) in Thais were comparable to ATV levels obtained from historical Caucasian cohorts using the standard dose of ATV/r $300 / 100 \mathrm{mg}$ QD [19]. We also observed a significant drop in total bilirubin concentrations and fewer incidents of hyperbilirubinemia among its study participants when ATV/r was used at 200/100 mg QD.

*Corresponding author: Anchalee Avihingsanon, MD, PhD, HIV-NAT, The Thai Red Cross AIDS Research Centre, 104 Ratchadamri Road, Pathumwan, Bangkok, Thailand 10330, Tel: + 66-2-255-7335; Fax: + 66-2-252-5779; E-mail: anchaleea2009@gmail.com

Received April 01, 2015; Accepted May 10, 2015; Published May 21, 2015

Citation: Avihingsanon A, Apornpong T, Kerr SJ, Make-a-nantawat W, Thammajaruk $\mathrm{N}$, et al. (2015) Low-Dose Ritonavir-Boosted Atazanavir (200/100 mg) Maintained a High Virological Efficacy Up To 4 Years in Treatment-Experienced HIV-1 Infected Adults: A Prospective Cohort Study from Asia. J AIDS Clin Res 6: 460 doi:10.4172/2155-6113.1000460

Copyright: (c) 2015 Avihingsanon A, et al. This is an open-access article distributed under the terms of the Creative Commons Attribution License, which permits unrestricted use, distribution, and reproduction in any medium, provided the original author and source are credited. 
Citation: Avihingsanon A, Apornpong T, Kerr SJ, Make-a-nantawat W, Thammajaruk N, et al. (2015) Low-Dose Ritonavir-Boosted Atazanavir (200/100 mg) Maintained a High Virological Efficacy Up To 4 Years in Treatment-Experienced HIV-1 Infected Adults: A Prospective Cohort Study from Asia. J AIDS Clin Res 6: 460. doi:10.4172/2155-6113.1000460

Page 2 of 6

However, there is no long-term efficacy data of low-dose ATV/r 200/100 mg QD for ARV-experienced Asian HIV-infected patients. This kind of information is imperative now that many patients are living longer on combination antiretroviral therapy (cART). Furthermore, since it is unlikely that a cure will be discovered any time soon, patients need to be on lifelong medication with good adherence. But for those who have already failed the first-line, a personalized second-line regimen may be required.

There is an increasing number of patients who are in need of second-line boosted protease inhibitor-based ART. Personalized medicine for these treatment-experienced patients in resource-limited settings (RLS) are less feasible because of costs and availability of the antiretroviral drugs through the countries' prospective healthcare program. As a result of this, $\mathrm{LPV} / \mathrm{r}$ is widely used for second-line treatment in RLS, but for patients with hyperlipidemia, atazanavir is a better alternative although the cost outweigh its usability. If long-term use of low-dose ATV/r 200/100 mg QD is safe and efficacious among ARV-experienced HIV-infected patients, this may provide patients in RLS an alternative, safer second-line regimen. Thus we assessed the long-term efficacy of low-dose ATV/r 200/100 mg QD for up to 6 years in $127 \mathrm{ARV}$-experienced, Asian HIV-infected patients.

\section{Patients and Methods}

ARV-experienced, HIV-infected adults on ATV/r 200/100 mg QD in combination with 2 NRTIs were followed in the prospective, longterm cohort (clinicaltrials.org\#NCT00411983) from January 2005 to March 2015 in Bangkok, Thailand. All subjects gave their consent to participate in this study. The study protocol for this cohort was approved by the Thailand Ministry of Public Health and the Chulalongkorn University Institutional Review Boards. Clinical data (i.e., adverse effects and toxicity, AIDS and non-AIDS events, reasons for stopping or switching ARV and use of concomitant medications) and laboratory data (CD4 counts, HIV-RNA, and other fasting safety parameters such as triglycerides, total cholestorol, glucose, liver and kidney function tests) were prospectively collected at six-month intervals.

\section{Atazanavir and ritonavir plasma concentrations}

Among those patients on standard dose of ATV/r, therapeutic drug monitoring (TDM) for plasma concentrations of atazanavir and ritonavir were performed prior and after switching to lower dose of ATV/r 200/100 mg QD plus 2 NRTIs. TDM for lower dose ATV/r was performed at least 1 month post-administration of the drugs. On the day TDM was performed, $5 \mathrm{~mL}$ of heparinized plasma samples were collected at $24 \mathrm{hr}$ post-drug intake (trough concentration: $C_{\text {trough }}$ ). Plasma concentrations of atazanavir and ritonavir were determined by a validated high-performance liquid chromatrography (HPLC) method at the HIV-NAT laboratory. Samples were centrifuged at 3,000 $\mathrm{g}$ for 10 min at $20^{\circ} \mathrm{C}$ and frozen at $<-20^{\circ} \mathrm{C}$ within 2 hours after collecting the blood.

\section{Statistical analyses}

Statistical analysis was carried out using Stata version 12 (Stata Corp LP, College Station, TX). Median (interquartile range [IQR]) and frequency (\%) were used to describe the patients' demographic characteristics for continuous and categorical data, respectively.

An 'intention-to-treat approach (ITT)' was used for all patients with at least one HIV-RNA measurement after baseline to evaluate the efficacy and safety of low-dose ATV/r. The proportion of patients with suppressed viral load (HIV RNA $<50$ copies/mL) at baseline and every 6 month intervals after initiating low-dose ATV/r were analyzed. $95 \%$ confidence interval $(95 \% \mathrm{CI})$ was also estimated. In this analysis subjects with missing data or who changed from ATV to another PI but were still under follow-up were imputed as failures. An 'on treatment (OT)' analysis was also conducted which included patients with a viral load measurement who were also taking ATV. The comparison of viral suppression was made by McNemar's test.

The change in safety lab parameters including CD4 counts, serum creatinine, estimated glomerular filtration rate (eGFR) by chronic kidney disease epidemiology formula (CKD-EPI), lipid profiles, and bilirubin including the prevalence of grade 3-4 hyperbilirubinemia (total bilirubin $>3.2 \mathrm{mg} / \mathrm{dl}$ ) prior to and after ATV/r 200/100 mg QD lower dose was analyzed by a paired t-test or Wilcoxon signed ranks test and chi-square tests as appropriate. The median IQR (25-75\%) and 95\%CI of ATV $C_{\text {trough }}$ were calculated. The proportion of patients who had ATV $C_{\text {trough }}$ levels $>0.15 \mathrm{mg} / \mathrm{L}$ was assessed. Statistical tests were 2 -sided and performed at a level of significance of 0.05 .

\section{Results}

\section{Demographics}

Baseline characteristics of the study population prior to using low-dose ATV/r are presented in Table 1 . A total of 127 patients $(50 \%$ female) switched to low-dose boosted ATV/r 200/100 mg once-daily. The median BMI was $21.9 \mathrm{~kg} / \mathrm{m}^{2}$ and the median BW was $60 \mathrm{~kg}$. All were ARV treatment-experienced with a history of NRTI and/or NNRTI failure(s) before boosted PIs were used. Almost half of the

\begin{tabular}{|c|c|}
\hline Characteristics & $N=127$ \\
\hline Female, $\mathrm{N}(\%)$ & $63(50)$ \\
\hline Median age; years (IQR) & $41(37-47)$ \\
\hline Subject older than 50 years old, N (\%) & $23(18)$ \\
\hline Median BMI (kg/m), IQR & $\begin{array}{c}21.9(19.6- \\
24.6)\end{array}$ \\
\hline Median weight $(\mathrm{BW})(\mathrm{kg}), \mathrm{IQR}$ & $60(50-67)$ \\
\hline Subject with BW> $60 \mathrm{~kg}, \mathrm{~N}(\%)$ & $55(48)$ \\
\hline $\begin{array}{l}\text { Regimen prior to switching to ATV/r 200/100, N (\%) } \\
\text { ATV/r } 300 / 100 \mathrm{mg} \\
\text { ATV } 400 \mathrm{mg} \\
\text { IDV/r } \\
\text { LPV/r } \\
\text { SQV/r } \\
\text { EFV or NVP failure }\end{array}$ & $\begin{array}{l}61(48) \\
8(6) \\
7(6) \\
37(29) \\
9(7) \\
5(4)\end{array}$ \\
\hline $\begin{array}{l}\text { Reasons for switching to ATV/r } 200 / 100 \\
\text { ATV plasma concentration }>0.85 \mathrm{mg} / \mathrm{L} \\
\text { Asymptomatic hyperbilirubinemia } \\
\text { Clinical Jaundice (symptomatic patients) } \\
\text { Other (pill burden from other boosted Pls, low BW, or reduced cost) }\end{array}$ & $\begin{array}{c}44(35) \\
20(16) \\
10(8) \\
53(42)\end{array}$ \\
\hline $\begin{array}{l}\text { Concomitant TDF } \\
\text { TDF } 300 \mathrm{mg} \text { alterative day } \\
\text { TDF } 300 \mathrm{mg} \text { once daily }\end{array}$ & $\begin{array}{l}118(93) \\
34(29) \\
84(71)\end{array}$ \\
\hline Median baseline CD4, cells/ $\mu \mathrm{L}$ (IQR) & $\begin{array}{c}486(368-696) \\
\text { ( range } 14- \\
1854)\end{array}$ \\
\hline Baseline HIV-RNA <50 copies/ml, N (\%) & $116(91.3)$ \\
\hline Median bilirubin, mg/dl (IQR) & $1.3(0.8-2.6)$ \\
\hline Median eGFR, $\mathrm{ml} / \mathrm{min} / 1.73 \mathrm{~m}^{2}(\mathrm{IQR})$ & $83(74-99)$ \\
\hline
\end{tabular}

IQR : interquatile range, ATV/r : atzanavir/ritonavir, IDV/r: indinavir/ritonavir, SQV/r saquinavir/ritonavir, LPV/r: lopinavir/ritonavir, EFV:efavirenz; NVP: nevirapine TDF: tenofovir disproxil fumarate; eGFR: estimated glomerular filtration rate Table 1: Baseline characteristics of 127 patients at time of initiating ATV/r 200/100 mg once daily. 
Citation: Avihingsanon A, Apornpong T, Kerr SJ, Make-a-nantawat W, Thammajaruk N, et al. (2015) Low-Dose Ritonavir-Boosted Atazanavir (200/100 mg) Maintained a High Virological Efficacy Up To 4 Years in Treatment-Experienced HIV-1 Infected Adults: A Prospective Cohort Study from Asia. J AIDS Clin Res 6: 460. doi:10.4172/2155-6113.1000460

Page 3 of 6

patients were previously on ATV/r 300/100 mg before switching to the lower dose ATV/r 200/100 mg. A total of $12(9.4 \%)$ patients had HIV RNA > 50 copies/mL when they started ATV/r lower dose; all of these patients have failed an NNRTI regimen but were PI-naïve. 93\% of them were using tenofovir disoproxil fumarate (TDF) concomitantly and $29 \%$ were using tenofovir $300 \mathrm{mg}$ every other day due to low eGFR. The main reasons for initiating lower dose ATV/r were: high ATV $C_{\text {trough }}>0.85 \mathrm{mg} / \mathrm{L}$ (35\%), asymptomatic hyperbilirubinemia (16\%), and clinical jaundice (symptomatic (8\%), and pill burden/lower BW/lower cost $(42 \%)$.

\section{Efficacy and safety}

The median duration of follow-up was 154 (IQR 65-271) weeks and maximum up to 482 weeks of follow-up. Sixty-four (50.4\%) and $40(31.5 \%)$ patients had been followed for more than 3 and 5 years, respectively. The percentage of patients followed for 24, 48 and 96 weeks were $90 \%, 77 \%$ and $68 \%$ respectively. The median CD 4 count increased significantly from 474 to 591 cells/ $\mu \mathrm{L}(P<0.001)$. The proportion of patients with plasma HIV-RNA $<50$ copies $/ \mathrm{mL}$ at each study visit is shown in Figure 1. During a median duration of 154 weeks of follow-up, $91.2 \%$ and $81.8 \%$ had HIV-RNA $<50$ copies/ $\mathrm{mL}$ when analyzed via ontreatment (OT) and intention-to-treat (ITT) approaches, respectively. Seven out of 10 patients with detectable viral load had low-level viremia (54-131 copies/mL). Another 3 cases had HIV RNA > 1000 copies $/ \mathrm{ml}$ due to self-discontinuation of ART 1-2 months prior to VL testing. For these patients, resistance testing was performed and none of them had any major PI mutations. ATV/r 200/100 mg was discontinued in all patients with detectable HIV RNA and standard dose of LPV/r or $\mathrm{ATV} / \mathrm{r}$ was initiated. We also looked at the predictors of response at 4 years. The variables included age, sex, body weight, body mass index, TDF use, baseline CD4, baseline HIV RNA, hepatitis B, hepatitis C and ATV concentration. In the multivariate analysis, none of these variables predicted the response at 4 years and only baseline HIV RNA was statistically significant in the univariate analysis (HR:1.57, 95\% CI:1.08-2.28, $\mathrm{p}=0.018$ ).

The data for safety parameters are summarized in Figure 2. The median (IQR) eGFR significantly improved after low-dose was initiated [eGFR 83 (IQR 71-98) to 88 (IQR 76-100) $\mathrm{ml} / \mathrm{min} / 1.73 \mathrm{~m}^{2}, \mathrm{p}<0.001$ ). For those 61 patients who used ATV/r 300/100 mg before they switched to ATV/r 200/100 mg, 59\% were exposed to IDV/r prior to taking ATV/r 300/100mg. The improvement of eGFR was greater among those who had never been exposed to IDV/r. The median eGFR before and after ATV/r 200/100 mg among those without IDV/r exposure was 81.5 (IQR 75.0-92.0) and $92.3(83.4-104.4) \mathrm{ml} / \mathrm{min} / 1.73 \mathrm{~m}^{2}, \mathrm{p}<0.001$,

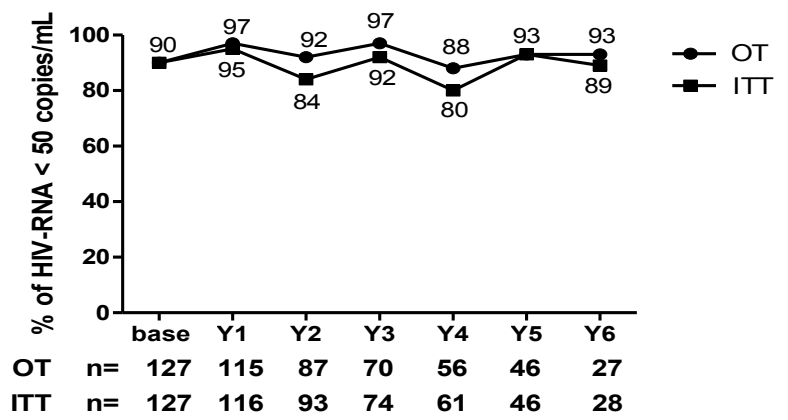

Figure 1: Proportion of patients with HIV-RNA $<50$ copies $/ \mathrm{mL}$ at time of ATV/r 200/100m mg initiation and every 6 months thereafter.

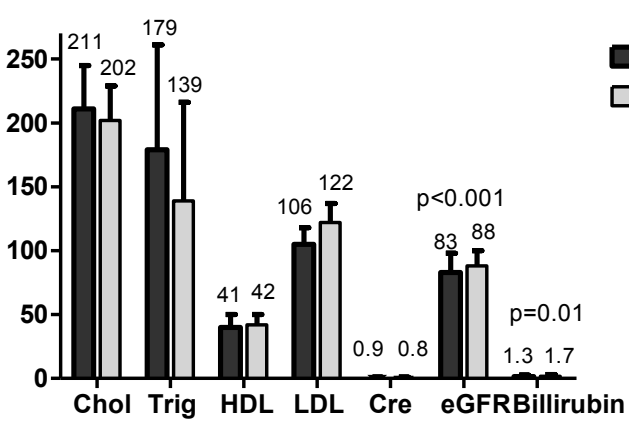

Cho=cholesterol, TG=triglyceride, cr=serum creatinine, eGFR=estimated glomerular filtration rate

Figure 2: The median (IQR) safety parameters at baseline (at time of ATV/r 200/100 mg initiation) and time of analysis (at last visit).

respectively. The median eGFR before and after ATV/r 200/100 mg among those with IDV/r exposure was 76.5 (IQR 65.2-85.4) and 80.5 (IQR $72.8-97.9) \mathrm{ml} / \mathrm{min} / 1.73 \mathrm{~m}^{2}, \mathrm{p}<0.001$, respectively. In addition, among those with prior IDV/r exposure, 4 of them had deteriorated renal function.

Overall median cholesterol, triglyceride, LDL and HDL did not statistically change before initiating low-dose ATV/r and at the last follow-up visit. However, median fasting cholesterol and triglyceride significantly improved after switching from LPV/r or IDV/r to low-dose $\mathrm{ATV} / \mathrm{r}$ [cholesterol 211 (IQR 194-245) to 182 (IQR 194-220), $\mathrm{p}=0.01$ and triglyceride 179 (IQR 117-261) to 122 (IQR 102-168), $\mathrm{p}=0.016$ ]. Patients on low-dose ATV/r 200/100 mg had a median bilirubin of 1.7 (IQR 1.2-3.0) mg/dl. For those patients on standard dose of ATV/r $300 / 100 \mathrm{mg}$ QD, the median bilirubin was 2.8 (IQR 1.6-4.0) and after switching to low-dose ATV/r 200/100 mg QD, the median bilirubin significantly dropped to 1.1 (IQR $0.6-1.8$ ) $\mathrm{mg} / \mathrm{dl}, \mathrm{p}=0.034 .9 .4 \%$ and $24.1 \%$ had grades $3-4$ hyperbilirubinemia (total bilirubin $>3.2 \mathrm{mg} / \mathrm{dl}$ ) when on ATV/r 200/100 mg and 300/100 mg respectively. Low-dose $\mathrm{ATV} / \mathrm{r}$ was well-tolerated in majority of the patients.

In $13(10.2 \%)$ patients, ATV/r 200/100mg QD was discontinued due to toxicity $(\mathrm{n}=10)$ and death $(\mathrm{n}=3)$. For toxicity, there were 4 patients with progressive $\mathrm{CKD}$, 2hepatitis $\mathrm{C}$ co-infected patients with elevated ALT grade 3, 1 patient with persistent jaundice, 1 patient with renal stones, 2 patients with acute pancreatitis, and 3 deaths. Causes of death were bacterial sepsis, end stage renal disease and cerebral aneurysm.

\section{Atazanavir plasma concentrations $\left(C_{\text {trough }}\right)$}

The median trough concentrations of atazanavir are plotted in Figure 3. All 42 patients using ATV/r 200/100 mg QD with available ATV $C_{\text {trough }}$ had atazanavir $C_{\text {trough }}>0.15 \mathrm{mg} / \mathrm{L}$ which is above the protein-binding adjusted IC 50 of PI-sensitive virus. In patients taking the ATV/r 200/100 mg dose, the median ATV concentrations were $0.6 \mathrm{mg} / \mathrm{L}$ (IQR $0.4-1.0 \mathrm{mg} / \mathrm{L}$ ) whereas for those on the standard dose ATV/r 300/100 mg, the median ATV concentrations were 1.0 (IQR 0.5-1.6) $\mathrm{mg}(\mathrm{p}=0.036)$ and $33 \%$ of patients on ATV/r 200/100 mg had atazanavir $\mathrm{C}_{\text {trough }}>0.85 \mathrm{mg} / \mathrm{L}$. In addition, all subjects with undetectable HIV-RNA $(\mathrm{N}=115)$ and detectable HIV-RNA $(\mathrm{N}=12)$ at time of lowdose ATV/r 200/100 mg initiation had atazanavir $\mathrm{C}_{\text {trough }}$ concentration $>0.180 \mathrm{mg} / \mathrm{L}$ and $0.30 \mathrm{mg} / \mathrm{L}$, respectively. The median (IQR) atazanavir concentrations for patients on the maintenance regimen and for those with detectable VL at time of $\mathrm{ATV} / \mathrm{r}$ lower dose initiation were 0.57 (0.37-1.18) $\mathrm{mg} / \mathrm{L}$ and $0.63(0.37-0.77) \mathrm{mg} / \mathrm{L}$, respectively. 
Citation: Avihingsanon A, Apornpong T, Kerr SJ, Make-a-nantawat W, Thammajaruk N, et al. (2015) Low-Dose Ritonavir-Boosted Atazanavir (200/100 mg) Maintained a High Virological Efficacy Up To 4 Years in Treatment-Experienced HIV-1 Infected Adults: A Prospective Cohort Study from Asia. J AIDS Clin Res 6: 460. doi:10.4172/2155-6113.1000460

Page 4 of 6

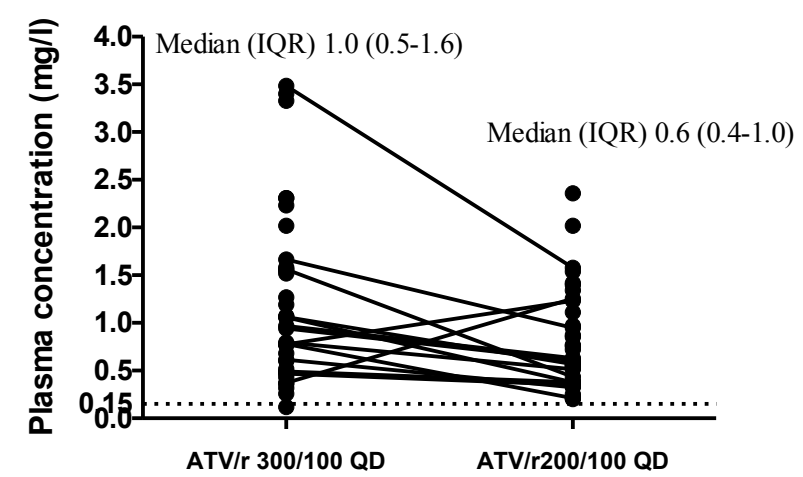

Figure 3: The plasma atazanavir concentration $\left(\mathrm{C}_{\text {trough }}\right.$ ) when used at $\mathrm{ATV} / \mathrm{r}$ 300/100 mg QD versus ATV/r 200/100 mg QD.

In this cohort, $93 \%$ of patients on ATV/r 200/100 mg QD were also on TDF; the median ATV concentrations were 0.55 (0.36-0.75) $\mathrm{mg} / \mathrm{L}$ for patients with TDF. There were three patients not on TDF treatment who had ATV concentrations of $1.34,2.02$, and $2.36 \mathrm{mg} / \mathrm{L}$. Amongst the patients on TDF, 20/39 (51\%) were taking TDF $300 \mathrm{mg}$ every alternate day due to low eGFR prior to initiating ATV/r 200/100 mg. The median (IQR) ATV concentrations were $0.55(0.36-0.75) \mathrm{mg} / \mathrm{L}$ and $0.54(0.38-0.97) \mathrm{mg} / \mathrm{L}$ for TDF standard dose and reduced dose, respectively. Of note, median ATV concentrations were not different between 24 women $(0.55$ (IQR $0.4-1.20) \mathrm{mg} / \mathrm{L})$ and $18 \mathrm{men}(0.58$ (IQR $0.4-0.8) \mathrm{mg} / \mathrm{L}), \mathrm{p}=0.81$.

\section{Discussion}

At present, long-term use of cART is necessary for lifelong HIV viral suppression and to prevent HIV disease progression. Given the long-term treatment, dose optimization for certain group is crucial to maintain good long-term outcome and to minimize the long-term side effects. A retrospective cohort study [18], and a pharmacokinetic study [19] conducted in Thai patients reported a good efficacy and optimal ATV concentrations when ATV/r 200/100 mg QD plus 2NRTIs were used. We then evaluated the long-term efficacy of ATV/r 200/100 mg based regimen in this study. The results showed that low-dose ATV/r 200/100 mg in 127 ARV treatment-experienced HIV-infected patients achieved high virological efficacy for up to 6 years of treatment even though, all patients had histories of NRTI and/or NNRTI failure in the past prior to boosted PI (including ATV/r 300/100 mg) use. Almost $96 \%$ of patients were on the standard dose (48\% with ATV/r 300/100 QD) PI based regimens and $4 \%$ on NNRTI at the time of low-dose ATV/r initiation. None of the patients had a history of PI failure. In this study, once-daily low-dose ATV/r at 200/100 mg with two NRTIs resulted in excellent antiviral efficacy with $91.2 \%$ of patients achieving and maintaining undetectable plasma HIV-RNA $(<50$ copies $/ \mathrm{mL})$ at a median follow-up of 154 weeks with one third displaying efficacy for more than 4 years. Interestingly, renal function and hyperbilirubinemia also improved after switching to the lower dose.

Lower BW of Thai patients may explain why the low dose ATV/r still has a high efficacy compared to Caucasians. Even among patients with $\mathrm{BW}>60 \mathrm{~kg}$, we observed that the low-dose was equally effective compared to the standard dose. This regimen was well-tolerated even though a few of the patients did switch to a different ARV due to the side effects. The high response rates in this cohort are consistent with other studies that have examined ATV/r 300/100 mg [5] and Saquinavir/r $1500 / 100 \mathrm{mg}$ [20]. Similarly, the efficacy results are consistent with other long-term studies for many PI-based cART [13,20,21].
All 42 patients with ATV Ctrough samples available were found to have adequate ATV trough concentrations. There has been some controversy whether TDF has an impact on ATV concentrations. Von Hentig et al. [1] reported that co-administration of TDF did not reduce the plasma concentrations of ATV whereas others [22,23] found TDF was associated with low ATV concentrations. In this cohort, only 3 subjects were taking non TDF regimen. These subjects had higher ATV Ctroughs than patients taking TDF, but because of very low numbers no statistical comparison was made.

Our PK findings in 24 females are consistent with previous reports that showed no clinical correlation between gender and atazanavir levels $[1,24]$. In contrast, Amadasi et al. [23] found that female patients tend to have higher atazanavir concentrations, but the most likely explanation for this discrepancy is that no adjustment was made for body weight as a confounding factor in their multivariate model. Previous studies have shown that higher trough concentrations of ATV may predispose the patients to elevated bilirubin concentrations, especially for ATV concentrations of $>0.85 \mathrm{mg} / \mathrm{L}$ which were associated with having a 3 -times higher risk for hyperbilirubinemia [10,25]. In this cohort, 33\% of the patients on ATV/r 200/100 mg had atazanavir $\mathrm{C}_{\text {trough }}>0.85 \mathrm{mg} / \mathrm{L}$.

Recently, Lescure et al. [26], proposed a new cut-off level for the concentration of ATV at 24 hours $\left(\mathrm{C}_{24}\right)$. They suggested that ATV $\mathrm{C}_{24}$ concentrations should be $0.180 \mathrm{mg} / \mathrm{L}$ for patients on maintenance therapy (undetectable plasma HIV-RNA at time of ATV/r initiation) and $0.30 \mathrm{mg} / \mathrm{L}$ for patients with detectable plasma HIV-RNA. In this cohort, all subjects with undetectable HIV-RNA and detectable HIVRNA at time of low-dose ATV/r 200/100 mg initiation had atazanavir $\mathrm{C}_{\text {trough }}$ concentrations $>0.180 \mathrm{mg} / \mathrm{L}$ and $0.30 \mathrm{mg} / \mathrm{L}$, respectively. These findings confirm that low-dose ATV/r 200/100 mg can provide adequate ATV concentrations if the patients have good ART adherence.

In previous intensive pharmacokinetic studies, 22 HIVinfected patients on low-dose ATV/r 200/100 mg had comparable pharmacokinetic profiles to Caucasians on standard dose ATV/r $300 / 100 \mathrm{mg}$ [19]. Having higher plasma concentrations of ATV may be a potential risk for acquiring arrhythmia in certain groups of people, particularly the elderly. Asymptomatic PR prolongation and ATV dosedependent QT interval prolongation have been observed particularly among. older patients who have many co-morbidities and therefore need to take multiple medications that can interact with boosted ATV. This can result in prolonged QTc and fatal arrhythmias, especially when ATV plasma concentrations are high. Furthermore, higher ATV plasma concentrations may contribute to renal stones $[7,8]$ and chronic kidney disease [9] as seen with boosted indinavir [11]. Interestingly, the median eGFR of our patients significantly improved after the dose of ATV/r was lowered to 200/100 mg QD. However, one third of the patients also took tenofovir dosed at $300 \mathrm{mg}$ every other day. Therefore, reducing the doses of both ATV/r and TDF may thwart the side effects of the kidney injury/damage among individuals at risk. The use of lower dose ATV/r 200/100 mg QD plus 2NRTIs for maintenance therapy may be an appropriate strategy for Thai/Asian ethnicities with undetectable HIV RNA.

The results from this study confirm the findings from a previous cohort study [18] conducted in 14 Thai patients who switched from other regimens to ATV/r 200/100 mg QD; all patients maintained their plasma HIV-RNA $<50$ copies/mL during the median follow-up of 112 weeks. This data provides some evidence that this regimen is adequate in HIV-infected patients in Thailand, at least for maintenance therapy among patients with undetectable VL. Whether this outcome applies to other ethnicities, particularly Caucasian is uncertain. 
Citation: Avihingsanon A, Apornpong T, Kerr SJ, Make-a-nantawat W, Thammajaruk N, et al. (2015) Low-Dose Ritonavir-Boosted Atazanavir (200/100 $\mathrm{mg}$ ) Maintained a High Virological Efficacy Up To 4 Years in Treatment-Experienced HIV-1 Infected Adults: A Prospective Cohort Study from Asia. J AIDS Clin Res 6: 460. doi:10.4172/2155-6113.1000460

Page 5 of 6

Previous studies in Thais showed that lower doses of other PIs had similar drug concentrations as those in Caucasians on standard dosages with sustained virological suppression and experienced fewer incidences of toxicities [14,27,28]. As a result of this, boosted PIs such as saquinavir/ $\mathrm{r}$ and indinavir/ $\mathrm{r}$ are currently prescribed at a lower dose than the dose stipulated by the pharmaceutical companies in the Thai clinical practice. Such findings have not been tested in nonAsians. Aside from having a lower body weight, factors such as genetic polymorphisms or differences in dietary consumption may account for the lower clearance when compared to Caucasians. However, this cohort demonstrated that lower dose of ATV is still effective in patients weighing more than $60 \mathrm{~kg}$.

As millions of Asians are being treated with the first-line antiretroviral therapy, it is expected that a substantial number will eventually also require second-line therapy. The cost of procuring second-line therapy is a major burden for countries with fragile economies. LPV/r is widely used for second-line treatment in resourcelimited settings including Thailand. Even though the long-term efficacy of LPV/r has been established but its frequent side effects of dyslipidemia and insulin resistance make the use of this ARV in the elderly or any patients with cardiovascular disease a little bit more cumbersome. In order to avoid complicated regimen for treatment options among the elderly and those with cardiovascular disease, a lipid friendly ARV is highly desirable. Thus, for patients developing hyperlipidemia, ATV may be a good but expensive alternative. In addition to an improved toxicity profile, a lower dose of ATV will significantly increase the accessibility to boosted PI-based second-line regimen for those who really need it. Hence, ATV/r 200/100 mg QD plus an appropriate backbone may serve as an ideal boosted PI alternative for secondline therapy, especially for maintenance therapy for patients with undetectable HIV-RNA after using the standard dose. A randomized study of the standard versus low-dose ATV/r is underway in Thailand (NCT01159223) to confirm the efficacy and tolerability of low-dose $\mathrm{ATV} / \mathrm{r}$ prior to its implementation nationally.

The study has several limitations. First, the sample size was relatively small. Second, it was not a randomized trial and last, it focused only on Thai patients. Therefore, our observed outcomes may not be applicable to other ethnicities, especially Caucasians. However a recent multicountry dose reduction study found reduced dose efavirenz was noninferior to standard doses [17]. Future studies should also include a pharmacogenetic component to identify which genetic polymorphisms are common across other Asian ethnicities. This information will substantially help improve the public health outcomes and alleviate the financial burdenin this region where a substantial number of people are infected with HIV.

In conclusion, a combination of low-dose ATV/r 200/100 mg once-daily and 2 NRTIs can achieve high virological efficacy in HIVinfected Asian patients who are sensitive to PIs for up to 4years. Use of low-dose ATV/r is highly beneficial for HIV-infected patients from resource limited settings because it will lower both the toxicities and costs, allowing more people access to the drug.

\section{Acknowledgements}

The authors are grateful to all of its participants and staff at HIV-NAT, Thai Red Cross AIDS Research Centre for their contributions.

Funding: This study was funded by the HIV-NAT, Thai Red Cross AIDS Research, National Health Security Office (NHSO), Bangkok, Thailand, The Thailand Research Fund (TRF), grant number RSA 5380002, the National Research Councils of Thailand (NRCT), grant number 2553-112 and The Aligning Care and Prevention of HIVIAIDS with Government Decentralization to Achieve Coverage and Impact: ACHIEVED Project (The Global fund Project,
Thailand), and the Royal College of Physicians of Thailand (RCPT), grant number $73 / 2552$.

Conflict of interest: KR has served as a consultant for Merck and Tibotec. $\mathrm{He}$ has had paid speaking engagements with Bristol-Meyers Squibb, Merck Roche, Jensen-Cilag, GlaxoSmithKline, and GPO. KR has also received Senior Researcher Scholar, Thai Research Fund (TRF); and the National Research University Project of $\mathrm{CHE}$ and the Ratchadaphiseksomphot Endowment Fund (HR1161A). All other authors declare no conflict of interest.

Author contributions: $\mathrm{AA}, \mathrm{SJK}, \mathrm{PP}, \mathrm{DMB}$, and $\mathrm{KR}$ developed the study protocol. AA, WM, SP, RAR, and AC conducted the study; recruited, enrolled, managed and cared for the patients. AA drafted the manuscript. TA performed all statistical analysis. NY performed all PK tests for the study. All authors have read the manuscript, provided feedback/comments and approved the final version of the manuscript.

\section{Clinical trial registration number: NCT00411983}

This study was partly presented at the Tenth International Congress on Drug Therapy in HIV Infection on 7-11 November 2010 at Glasgow, UK. Abstract No P60.

\section{References}

1. von Hentig N, Dauer B, Haberl A, Klauke S, Lutz T, et al. (2007) Tenofovir comedication does not impair the steady-state pharmacokinetics of ritonavirboosted atazanavir in HIV-1-infected adults. Eur J Clin Pharmacol 63: 935-940.

2. Colonno R, Rose R, McLaren C, Thiry A, Parkin N, et al. (2004) Identification of $150 \mathrm{~L}$ as the signature atazanavir (ATV)-resistance mutation in treatment-naive HIV-1-infected patients receiving ATV-containing regimens. J Infect Dis 189: 1802-1810.

3. Johnson M, Grinsztejn B, Rodriguez C, Coco J, DeJesus E, et al. (2006) 96week comparison of once-daily atazanavir/ritonavir and twice-daily lopinavir/ ritonavir in patients with multiple virologic failures. AIDS 20: 711-718.

4. Molina JM, Andrade-Villanueva J, Echevarria J, Chetchotisakd P, Corral J, et al. (2008) Once-daily atazanavir/ritonavir versus twice-daily lopinavir/ritonavir, each in combination with tenofovir and emtricitabine, for management of antiretroviral-naive HIV-1-infected patients: 48 week efficacy and safety results of the CASTLE study. Lancet 372: 646-655.

5. Molina JM, Andrade-Villanueva J, Echevarria J, Chetchotisakd P, Corral J, et al. (2010) Once-daily atazanavir/ritonavir compared with twice-daily lopinavir/ ritonavir, each in combination with tenofovir and emtricitabine, for management of antiretroviral-naive HIV-1-infected patients: 96-week efficacy and safety results of the CASTLE study. J Acquir Immune Defic Syndr 53: 323-32.

6. Squires K, Lazzarin A, Gatell JM, Powderly WG, Pokrovskiy V, et al. (2004) Comparison of once-daily atazanavir with efavirenz, each in combination with fixed-dose zidovudine and lamivudine, as initial therapy for patients infected with HIV. J Acquir Immune Defic Syndr 36: 1011-1019.

7. Anderson PL, Lichtenstein KA, Gerig NE, Kiser JJ, Bushman LR (2007) Atazanavir-containing renal calculi in an HIV-infected patient. AIDS 21: 10601062.

8. Moriyama Y, Minamidate $\mathrm{Y}$, Yasuda M, Ehara H, Kikuchi M, et al. (2008) Acute renal failure due to bilateral ureteral stone impaction in an HIV-positive patient. Urol Res 36: 275-277.

9. Mocroft A, Kirk O, Reiss P, De Wit S, Sedlacek D, et al. (2010) Estimated glomerular filtration rate, chronic kidney disease and antiretroviral drug use in HIV-positive patients. AIDS 24: 1667-1678.

10. Colombo S, Buclin T, Cavassini M, Décosterd LA, Telenti A, et al. (2006) Population pharmacokinetics of atazanavir in patients with human immunodeficiency virus infection. Antimicrob Agents Chemother 50: 3801 3808.

11. Collin F, Chêne G, Retout S, Peytavin G, Salmon D, et al. (2007) Indinavir trough concentration as a determinant of early nephrolithiasis in HIV-1-infected adults. Ther Drug Monit 29: 164-170.

12. Patel AK, Patel KK, Patel JK, Sharma RL, Ranjan RR (2006) Effectiveness of low-dose indinavir/ritonavir at $400 / 100 \mathrm{mg}$ twice a day with 2 nucleoside reverse transcriptase inhibitors in nonnucleoside reverse transcriptase inhibitor-experienced HIV-infected patients in India: 1-year follow-up. J Acquir Immune Defic Syndr 43:123-126.

13. Mootsikapun $P$, Chetchotisakd P, Anunnatsiri S, Boonyaprawit $P$ (2005) Efficacy and safety of indinavir/ritonavir $400 / 100 \mathrm{mg}$ twice daily plus two nucleoside analogues in treatment-naive HIV-1-infected patients with CD4+ T-cell counts $<200$ cells/mm3: 96-week outcomes. Antivir Ther 10: 911-916. 
Citation: Avihingsanon A, Apornpong T, Kerr SJ, Make-a-nantawat W, Thammajaruk N, et al. (2015) Low-Dose Ritonavir-Boosted Atazanavir (200/100 $\mathrm{mg}$ ) Maintained a High Virological Efficacy Up To 4 Years in Treatment-Experienced HIV-1 Infected Adults: A Prospective Cohort Study from Asia. J AIDS Clin Res 6: 460. doi:10.4172/2155-6113.1000460

Page 6 of 6

14. Boyd M, Mootsikapun P, Burger D, Chuenyam T, Ubolyam S, et al. (2005) Pharmacokinetics of reduced-dose indinavir/ritonavir 400/100 $\mathrm{mg}$ twice daily in HIV-1-infected Thai patients. Antivir Ther 10: 301-307.

15. Cressey TR, Leenasirimakul P, Jourdain G, Tod M, Sukrakanchana PO, et al. (2005) Low-doses of indinavir boosted with ritonavir in HIV-infected Thai patients: pharmacokinetics, efficacy and tolerability. J Antimicrob Chemother 55: 1041-1044

16. Wasmuth JC, Rodermann E, Voigt E, Vogel M, Lauenroth-Mai E, et al. (2007) Comparison of indinavir + ritonavir $600+100 \mathrm{mg}$ vs. $400+100 \mathrm{mg}$ BID combinations in HIV1-infected patients guided by therapeutic drug monitoring. Eur J Med Res 12: 289-294.

17. ENCORE Study Group, Puls R, Amin J, Losso M, Phanuphak P, et al. (2014) Efficacy of $400 \mathrm{mg}$ efavirenz versus standard $600 \mathrm{mg}$ dose in HIV-infected, antiretroviral-naive adults (ENCORE1): a randomised, double-blind, placebocontrolled, non-inferiority trial. Lancet 383: 1474-1482.

18. Chetchotisakd P, Anunnatsiri S (2008) Low-dose, once-daily atazanavir/ ritonavir (200/100): an effective treatment for HIV-infected patients in Thailand J Acquir Immune Defic Syndr 49: 230-231.

19. Avihingsanon A, van der Lugt J, Kerr SJ, Gorowara M, Chanmano S, et al. (2009) A low dose of ritonavir-boosted atazanavir provides adequate pharmacokinetic parameters in HIV-1-infected Thai adults. Clin Pharmacol Ther 85: 402-408.

20. Ananworanich J, Gayet-Ageron A, Ruxrungtham K, Chetchotisakd P, Prasithsirikul W, et al. (2008) Long-term efficacy and safety of first-line therapy with once-daily saquinavir/ritonavir. Antivir Ther 13: 375-380.

21. Mills AM, Nelson M, Jayaweera D, Ruxrungtham K, Cassetti I, et al. (2009)
Once-daily darunavir/ritonavir vs. lopinavir/ritonavir in treatment-naive, HIV-1infected patients: 96-week analysis. AIDS 23: 1679-1688.

22. Taburet AM, Piketty C, Chazallon C, Vincent I, Gérard L, et al. (2004) Interactions between atazanavir-ritonavir and tenofovir in heavily pretreated human immunodeficiency virus-infected patients. Antimicrob Agents Chemother 48 2091-2096.

23. Amadasi S, Odolini S, Foca E, Panzali A, Cerini C, et al. (2013) Evaluation of boosted and unboosted atazanavir plasma concentration in HIV infected patients. Curr HIV Res 11: 642-646.

24. Avihingsanon A, Kerr SJ, Punyawudho B, van der Lugt J, Gorowara M, et al. (2013) Short communication: Aging not gender is associated with high atazanavir plasma concentrations in Asian HIV-infected patients. AIDS Res Hum Retroviruses 29: 1541-1546.

25. Smith DE, Jeganathan S, Ray J (2006) Atazanavir plasma concentrations vary significantly between patients and correlate with increased serum bilirubin concentrations. HIV Clin Trials 7: 34-38.

26. Lescure FX, Poirier JM, Meynard JL, Guiard-Schmid JB, Zouai O, et al. (2010) Factors predictive of virological failure on atazanavir in $310 \mathrm{HIV}$-infected patients. AIDS 24: 1593-1595.

27. Autar RS, Boffito M, Hassink E, Wit FW, Ananworanich J, et al. (2005) Interindividual variability of once-daily ritonavir boosted saquinavir pharmacokinetics in Thai and UK patients. J Antimicrob Chemother 56: 908 913.

28. van der Lugt J, Autar RS, Ubolyam S, Garcia EF, Sankote J, et al. (2008) Pharmacokinetics and short-term efficacy of a double-boosted protease inhibitor regimen in treatment-naive HIV-1-infected adults. J Antimicrob Chemother 61: 1145-1153. 